\title{
INCUBACIÓN DE HUEVOS Y DETERMINACIÓN DEL CICLO BIOLÓGICO DE Chironomus sp. (CHIRONOMIDAE, DÍPTERA) EN CUBETAS DE PLÁSTICO
}

\author{
Germán MURRIETA MOREY ${ }^{1}$, Jorge NÁJAR², Fernando ALCÁNTARA ${ }^{3}$ \\ 1 Instituto Nacional de Pesquisas da Amazônia, Laboratório de Parasitologia de Peixes Av. André Araújo 2936, Petrópolis, \\ CEP: 69.067-375, Manaus, Amazonas, Brasil. E-mail: germantiss@hotmail.com \\ 2 Universidad Nacional de la Amazonía Peruana. Plaza Serafín Filomeno s/n. Iquitos. Perú. \\ 3 Instituto de Investigaciones de la Amazonía Peruana. Programa de Investigación para el Uso y Conservación del Agua y \\ sus Recursos. Carretera Iquitos Nauta. Km. 4.5. Iquitos. Perú.
}

\section{RESUMEN}

Este estudio fue realizado en el Centro de Investigaciones de Quistococha, en Iquitos, Perú, con la finalidad de determinar la influencia de la disponibilidad de oxígeno disuelto durante la producción de larvas y el ciclo biológico del Chironomus sp., en incubación en ambiente artificial. Se colectaron sacos ovígeros de Chironomus sp. en los bordes de los tanques de producción de alimento vivo y la incubación fue realizada con aireación y sin aireación. En la incubación con aireación se obtuvo un número promedio de 491 larvas, mientras que sin aireación el número fue de 23. Esto evidencia la necesidad de la aireación con fines de incrementar los niveles de oxígeno disuelto en el agua y una mayor sobrevivencia de las larvas. El ciclo biológico de los quironómidos en estudio se prolongó durante 14 días, desde el inicio de la incubación de los huevos hasta la eclosión. Las larvas son visibles a simple vista a los cuatro días. Las larvas pasan por cuatro estadios durante ocho días y luego se transforman en pupas, estadio en el que permanecen durante dos días para dar origen a los adultos. El método de colección de sacos ovígeros y de incubación desarrollado es relativamente sencillo y puede ser aplicado a mayor escala con fines de producción de alimento vivo para las fases larvales de peces.

PALABRAS CLAVE: Quironómidos, producción de larvas, Amazonía peruana.

\section{EGG INCUBATION AND DETERMINATION OF THE BIOLOGICAL CYCLE OF Chironomus sp., (CHIRONOMIDAE, DIPTERA) IN PLASTIC BUCKETS}

\begin{abstract}
This work was developed at the Quistococha Research Center in Iquitos, Peru, in order to determine the influence of the availability of dissolved oxygen during larvae production and the life cycle of Chironomus sp., in artificial environment. The eggs of Chironomus sp. were collected at the edge of the tanks of live food production and these were incubated in plastic buckets with aeration and without aeration. We obtained 491 larvae in the incubation with aeration, while only 23 lavae were obtained without aeration. This demonstrates the need for aeration in order to increase levels of dissolved oxygen in the water and a better survival of the larvae. The life cycle of non-biting midges lasted 14 days from the start of incubation of eggs to the hatch. The larvae hatched within four days, passed through four larval stages during eight days; then they turned into pupae stage that lasted two days to then give rise to the adults. The developed method is relatively simple and can be applied to produce living food at large scale for fish larvae.
\end{abstract}

KEYWORDS: non-biting midge, larvae production, Peruvian Amazon. 


\section{INTRODUCCIÓN}

Los dípteros de la familia Chironomidae son comúnmente conocidos como mosquitos no picadores, debido a la reducción de las piezas bucales y a que no se alimentan durante la fase de adultos. Estos insectos están ampliamente distribuidos en el mundo, ocurren en ambientes de agua salada y agua dulce, y son frecuentemente más abundantes en agua dulce. A nivel mundial se estima que existen más de 15,000 especies y representan más del $50 \%$ del número total de macro invertebrados registrados (Coffman et al., 2008).

El corto ciclo de vida y las altas densidades de estos dípteros han favorecido la ejecución de numerosos estudios sobre la productividad y dinámica de sus poblaciones. En particular, la familia Chironomidae, juega un papel muy importante en el procesamiento del detritus y en la interacción de los ciclos tróficos en aguas lénticas y lóticas (Armitage, 1995).

Según Apperson et al. (2006), existen cuatro estadios del ciclo de vida de los quironómidos denominados instar. Los huevos son depositados en la superficie del agua en masas gelatinosas que contienen aproximadamente 600 huevos. Los huevos se precipitan al fondo de los cuerpos de agua y se incuban por unos días, para después producir las larvas que se entierran en el lodo o en el material orgánico, construyendo pequeños tubos en los cuales viven, aumentando su tamaño a medida que van creciendo. El material orgánico suspendido en el agua y el material de fondo es utilizado como alimento para el desarrollo de la larva. Gradualmente, las larvas van cambiando su color de rosado a rojo oscuro, por lo que son llamadas "gusanos de sangre". El color rojo resulta del alto contenido en hierro presente en la hemoglobina que permite respirar a las larvas cuando las concentraciones de oxígeno en el agua son muy bajas. Los estadios larvales pueden durar menos de dos a siete semanas, dependiendo de la temperatura del agua, con estadios cortos en climas tropicales con temperaturas entre $21^{\circ}$ a $26^{\circ} \mathrm{C}$ y largos en climas fríos con temperaturas entre $0^{\circ}$ a $10^{\circ} \mathrm{C}$. Las larvas se transforman en pupas mientras permanecen en sus tubos. Luego de tres días, las pupas nadan activamente a la superficie, transformándose en adultos en unas horas. Los adultos buscan aparearse al cabo de pocas horas, porque no se alimentan y tan sólo viven de tres a cinco días (Armitage, 1995).
Con el crecimiento de la pesca como una actividad deportiva, además del impulso y gran desarrollo actual de la Acuicultura en el mundo entero, estos insectos se han convertido en una de las fuentes más utilizadas para alimentar a los peces y han despertado el interés en la acuariofilia. Las larvas de quironómidos son una excelente fuente de proteína (De La Noue \& Choubert, 1985), lípidos, vitaminas y minerales (Mclarney et al., 1974). Habib et al. (1997) mencionan que en las prácticas de acuicultura se alimenta a los peces y camarones con larvas de quironómidos como fuente de minerales. Es así, que países como Hong Kong y Tailandia se han convertido en grandes productores de quironómidos, desarrollando un mercado a escala global (Armitage, 1995).

El valor de los quironómidos fue estudiado por Sudgen (1973), que reportó un porcentaje proteico de $56 \%$ para las larvas. El alto contenido proteico, la alta digestibilidad representada por el $73.6 \%$, como menciona De La Noue \& Choubert (1985) y la aparente función en pequeñas cantidades como promotor de crecimiento en dietas de peces y crustáceos, hacen de las larvas de quironómidos una fuente rica de alimento para organismos de cría. Asimismo, Armitage (1995) sugiere el uso de larvas y pupas de quironómidos como fuente de alimento debido a su alto contenido nutritivo; contienen $86 \%$ de humedad, 48 a $55 \%$ de proteína, $14 \%$ de lípidos, $23 \%$ de carbohidratos, $4 \%$ de quitina, $9 \%$ de ceniza, y con niveles de energía de 4.1 a $6.1 \mathrm{~K} \mathrm{Cal} \mathrm{g}^{-1}$.

El objetivo de este estudio es determinar el ciclo de vida y la producción de larvas de Chironomus sp. a través de la incubación de huevos en condiciones experimentales en la Amazonía peruana.

\section{MATERIAL Y MÉTODOS}

Se colectaron sacos ovígeros de aspecto gelatinoso con huevos de quironómidos de las unidades de producción masiva de alimento vivo de las instalaciones del Centro de Investigaciones de Quistococha del Instituto de Investigaciones de la Amazonía Peruana en Iquitos, durante el mes de Octubre del 2011.

Para la colección se utilizó un vaso de precipitación de $250 \mathrm{cc}$, con el cual se desprendieron las masas de huevos adheridas a las paredes de las unidades de producción, cerca de la película superficial. Las masas de huevos colectadas fueron depositadas en una bandeja de plástico para su traslado al laboratorio. 
Se utilizaron ocho envases de plástico de $1.5 \mathrm{~L}$ de capacidad como unidades de incubación, colocándoles $1 \mathrm{~L}$ de agua, $250 \mathrm{ml}$ de "agua verde" y 1 $\mathrm{g}$ de harina de pescado por cada unidad.

La incubación fue realizada en dos tratamientos con cuatro repeticiones y la distribución fue efectuada en un diseño completamente al azar. Cuatro de las unidades estuvieron provistas de aireación suministrada por piedras difusoras conectadas a una pequeña bomba de aireación eléctrica (Figura 1), y cuatro unidades no contaron con aireación.

En cada unidad fue colocado un saco ovígero asumiendo que tenían un peso y número equivalente de huevos. Las unidades fueron expuestas a 12 horas de luz y 12 horas de oscuridad. Se utilizó como alimento micro algas disponibles en el inóculo de "agua verde" con dominancia de Chlorella sp. y Scenedesmus cuadricauda. La harina de pescado fue suministrada como fertilizante del agua para la producción de organismos planctónicos. Las larvas logradas de cada masa de huevos fueron colectadas con pipetas y contadas en placas Petri al término del período de incubación.

Diariamente se determinaron los principales parámetros físico-químicos del agua por un periodo de doce días, como se indica: temperatura, oxígeno disuelto y $\mathrm{pH}$. El amonio y los nitritos fueron determinados al sexto día de incubación. La temperatura fue determinada con un termómetro Fisher de máxima y mínima, el oxígeno disuelto con un oxímetro digital YSI, el $\mathrm{pH}$ con un $\mathrm{pH}$ meter digital y el amonio y los nitritos fueron determinados mediante un kit de La Motte.

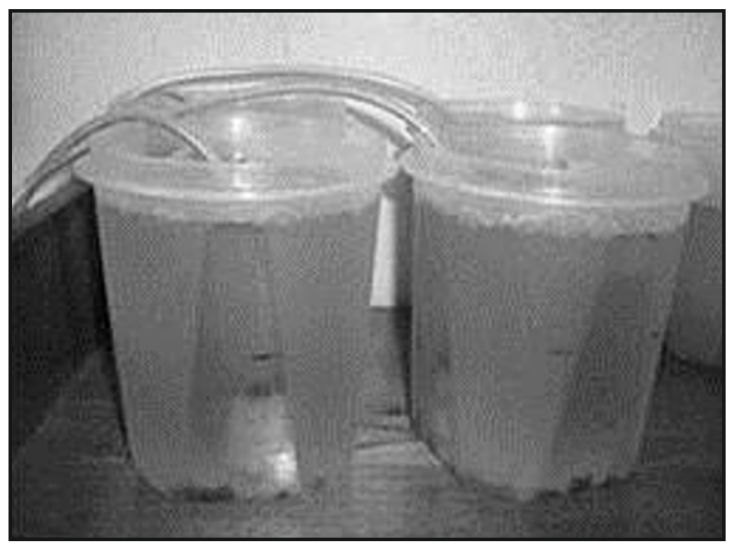

Figura 1. Incubadoras de sacos ovígeros de Chironomus sp. con aireación.

\section{RESULTADOS}

Los sacos ovígeros de Chironomus sp. estudiados tienen la forma de una coma y están compuestos por masas gelatinosas transparentes con numerosos huevos de forma oval dispuestos en espiral a lo largo del saco (Figura 2). Luego de la eclosión, las larvas permanecieron en el fondo del ambiente acuático y a través de su crecimiento pasaron por cuatro estadios o instar, cada uno con sus características propias. Se obtuvo $491.00 \pm 62.44$ larvas en el sistema con aireación y $23.25 \pm 13.76$ larvas en el sistema sin aireación (Tabla 1). Estos valores fueron significativamente diferentes (prueba $\left.\mathrm{F}_{(1,6)}=53.52, p<0.001\right)$. Durante su estadio larval, las larvas formaron tubos en el fondo con el material orgánico presente, el cual lo utilizaron como refugio y fuente de alimento.

La duración total del ciclo biológico de Chironomus sp. en este estudio fue de 14 días, contados a partir del momento de colección de los sacos ovígeros. Luego de la eclosión, las larvas permanecieron en el fondo de las unidades experimentales, siendo visibles a simple vista a partir del cuarto día. En el estadio de larva, éstas tenían color rojo intenso y observamos la presencia de un penacho con finos apéndices en el dorso del primer y segundo segmento torácico (Figura 3). En el décimo segundo día, las larvas pasaron al estadio de pupa de color rojo oscuro intenso en forma de coma y con dos penachos de apéndices finos en el dorso, semejantes a una pluma. (Figura 4). En el estadio de pupa permanecieron por dos días, luego de lo cual alcanzaron el estadio de adulto (Figura 5).

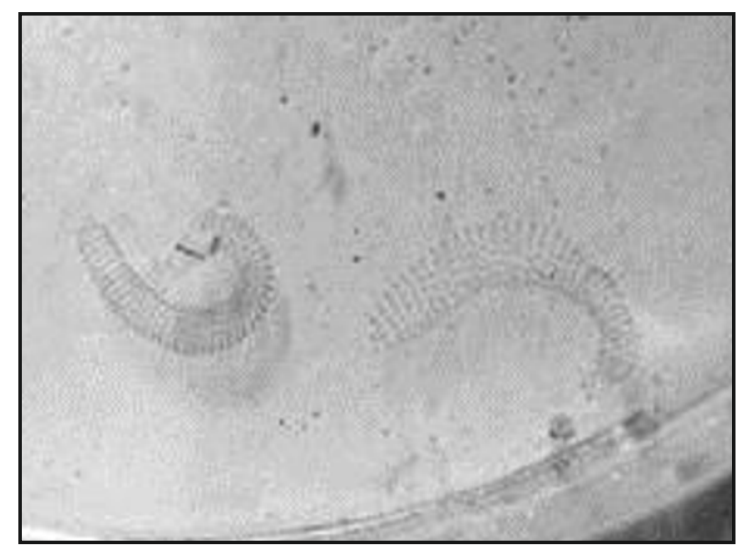

Figura 2. Sacos ovígeros de huevos de Chironomus sp. 
Observamos diferencias en la calidad de agua de las unidades de cultivo, en cuanto se refiere a los niveles de oxígeno disuelto (media \pm error típico): $6.35 \pm 0.02 \mathrm{mg} . \mathrm{L}^{-1}$ con aireación y $1.07 \pm 0.04 \mathrm{mg} . \mathrm{L}^{-1}$ sin aireación (Tabla 2). En cuanto a la temperatura y el $\mathrm{pH}$, fueron similares en ambas condiciones con valores promedios de $26^{\circ} \mathrm{C}$ y 7.5 , respectivamente. Los niveles de nitrógeno amoniacal fueron altos $(>$ 3,0 mg. $\left.\mathrm{L}^{-1}\right)$, mientras que los niveles de nitrito fueron bajos $\left(<0.05 \mathrm{mg} . \mathrm{L}^{-1}\right)$ en ambas condiciones.

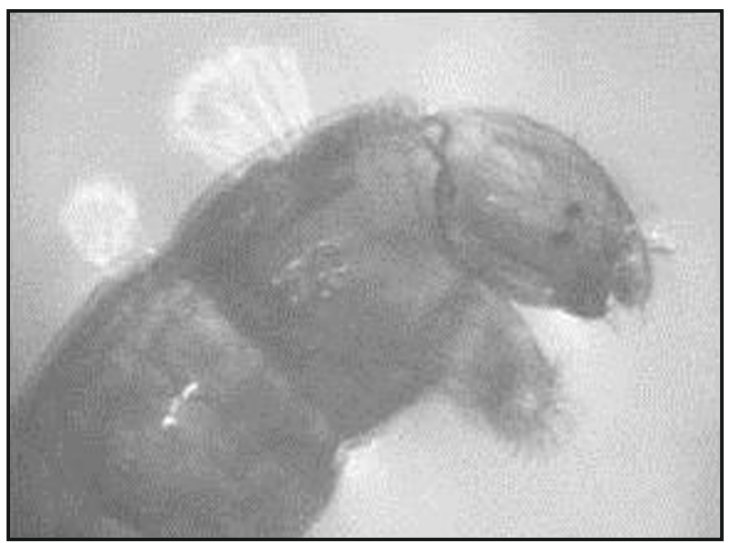

Figura 3. Larva de Chironomus sp.

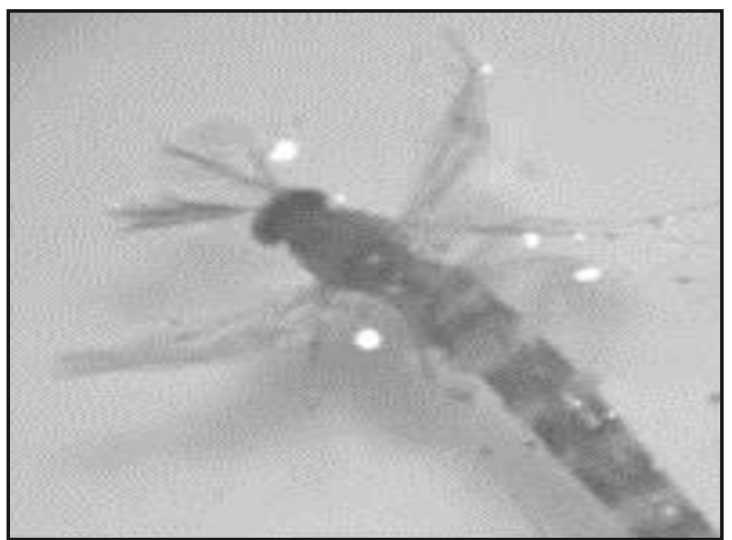

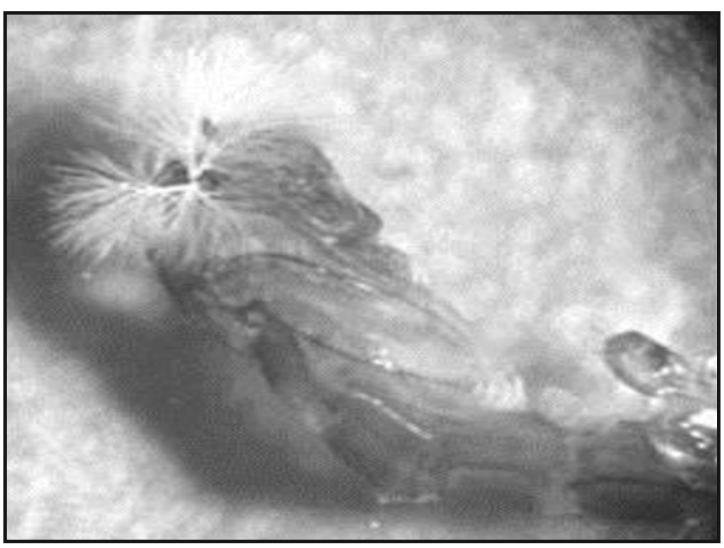

Figura 4. Pupa de Chironomus sp.

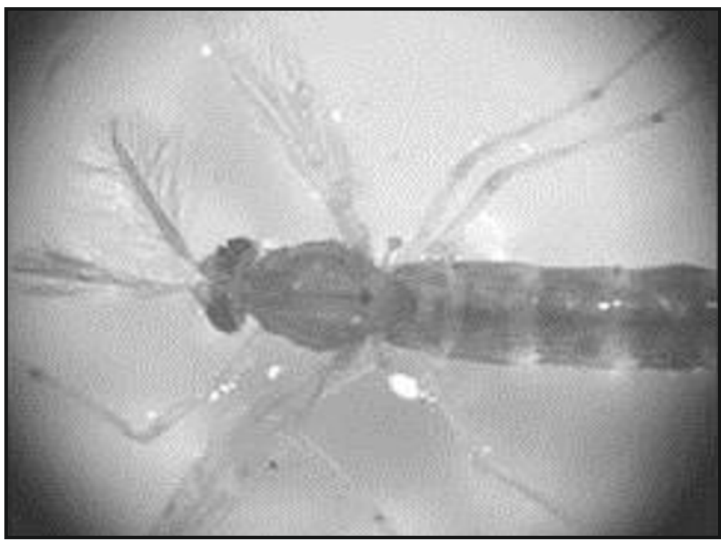

Figura 5. Individuo adulto de Chironomus sp

Tabla 1. Producción de larvas de Chironomus sp. por incubación de huevos en cubetas de plástico con y sin aireación.

\begin{tabular}{c|c|c|c|c|c}
\hline \multirow{2}{*}{ Tratamiento } & \multicolumn{4}{|c|}{ Número de larvas producidas } & \multirow{2}{*}{ Promedio \pm error } \\
\cline { 2 - 5 } & Cubeta 1 & Cubeta 2 & Cubeta 3 & Cubeta 4 & \\
\hline Con aireación & 655 & 409 & 380 & 520 & $491.00 \pm 62.44$ \\
Sin aireación & 0 & 36 & 56 & 1 & $23.25 \pm 13.76$ \\
\hline
\end{tabular}


Tabla 2. Calidad de agua de las cubetas de incubación de huevos de quironómidos con aireación y sin aireación.

\begin{tabular}{clccc}
\hline Tratamiento & & $\begin{array}{c}\text { Oxígeno disuelto } \\
(\mathbf{m g . L - 1})\end{array}$ & $\begin{array}{c}\text { Temperatura } \\
\left({ }^{\circ} \mathbf{C}\right)\end{array}$ & pH \\
\hline Con aireación & Media & 6.35 & 26.4 & 7.5 \\
& N & 12 & 12 & 12 \\
& Desv. típica & 0.08 & 0.1 & 0.0 \\
& Mínimo & 6.2 & 26.2 & 7.5 \\
& Máximo & 6.5 & 26.6 & 7.5 \\
& Error típico & 0.02 & 0.03 & 0.0 \\
\hline \multirow{3}{*}{ Sin aireación } & Media & 1.07 & 26.3 & 7.5 \\
& N & 12 & 12 & 12 \\
& Desv. típica & 0.1 & 0.1 & 0.04 \\
& Mínimo & 0.8 & 26.2 & 7.4 \\
& Máximo & 1.2 & 26.6 & 7.6 \\
& Error típico & 0.04 & 0.04 & 0.01 \\
\hline
\end{tabular}

Tabla 3. Duración en días de cada fase de desarrollo en diferentes especies de Chironomus incubadas a diferentes temperaturas

\begin{tabular}{|c|c|c|c|c|c|c|}
\hline \multirow{2}{*}{ Especie } & \multicolumn{4}{|c|}{ Duración de fase de desarrollo (días) } & \multirow{2}{*}{ Temperatura } & \multirow{2}{*}{$\begin{array}{c}\text { Referencia } \\
\text { bibliográfica }\end{array}$} \\
\hline & Huevo & Larva & Pupa & Total & & \\
\hline Chironomus sp. & 4 & 8 & 2 & 14 & $26^{\circ} \mathrm{C}$ & Presente estudio \\
\hline C. strenzkei & 2 & 6 & 2 & 10 & $26^{\circ} \mathrm{C}$ & Fittkau, 1965; Danks, 2006 \\
\hline C. xanthus & 2 & 9 & 2 & 13 & $24^{\circ} \mathrm{C}$ & Fonseca \& Rocha, 2004 \\
\hline C. xanthus & 3 & 10 & 2 & 15 & $250 \mathrm{C}$ & Strixino \& Trivinho-Strixino 1985 \\
\hline C. calligrafus & $5 \pm 1.2$ & $19 \pm 3$ & $10 \pm 3$ & $23(18-28)$ & $25^{\circ} \mathrm{C}$ & Florencia et al., 2008 \\
\hline C. riparius & 5 & 15 & 5 & 25 & $20^{\circ} \mathrm{C}$ & Jackson \& Sweeney, 1995 \\
\hline
\end{tabular}

\section{DISCUSIÓN}

\section{PRODUCCIÓN DELARVAS}

En el presente estudio mostramos un alto rendimiento en la producción de larvas de Chironomus sp. a través de la incubación de sacos ovígeros en cubetas plásticas con aireación. La mejor respuesta conseguida con la aplicación de aireación demuestra la necesidad de oxígeno para el desarrollo embrionario y fases iniciales de las larvas. Trivinho-Strixino \& Strixino (1982) reportan una producción de 500 a 1045 larvas por saco ovígero para Chironomus xanthus, que son valores mayores a los presentados en este estudio (491 larvas), lo que podría deberse a un aspecto particular de la especie.

Los niveles de temperatura y $\mathrm{pH}$ en las unidades experimentales en nuestro estudio fueron semejantes en los dos tratamientos, deduciéndose que el uso de la harina de pescado y del inóculo como fuentes de nutrientes no altera estas variables en los ambientes de cría. Además, podríamos decir que se otorgaron en cantidades adecuadas, permitiendo la eclosión, sobrevivencia y crecimiento de Chironomus sp. hasta alcanzar el estadio de adulto.

Mason (1973), Coffman \& Ferrington (1979) y Pinder (1986), reportaron que la profundidad del ambiente, junto con los valores elevados de temperatura, favorecen el incremento en la actividad fotosintética por ser muy pequeña la columna de agua y estar disponibles los nutrientes necesarios para la productividad acuática. Esta situación propicia una mayor disponibilidad de alimento para los quironómidos debido a que la mayoría se 
alimenta del detritus generado a partir del alimento producido en la superficie o del generado en el mismo sedimento. Asimismo, Navarrete et al. (2004) señalan que los parámetros más importantes para la abundancia de los quironómidos son la concentración de oxígeno, la dureza del agua, conductividad, transparencia y $\mathrm{pH}$, señalando que este modelo da una mayor certeza de los parámetros más relacionados con la abundancia de estos insectos, dado que en la naturaleza los organismos responden al efecto conjunto de diversos factores ambientales y no a uno solo.

Aunque numerosos estudios reportan la importancia de los residuos orgánicos en la dieta de los quironómidos, los componentes nutricionales no-vivos han sido discutidos, ya que mucho del material consumido no es digerido (celulosa, lignina y cenizas) y rápidamente pasa por el intestino. Por otra parte, los microorganismos tienen alto valor nutricional y pueden ser importantes en la síntesis de vitaminas esenciales que son utilizadas por los quironómidos (Fonseca \& Rocha, 2004). Microorganismos como bacterias, hongos y protozoos pueden ser utilizados como alimento de los quironómidos o pueden servir como insumos para transformar los residuos en nutricionales y formas consumibles (Mclachlan et al., 1979; Ward \& Williams, 1986; Berg, 1995). Según Hinton (1980), y Pinder (1995), la masa gelatinosa posee también numerosos carbohidratos que se constituyen como soporte para las larvas durante la fase de dispersión. Las larvas abandonan la masa gelatinosa y permanecen planctonófagas, hasta que encuentran el hábitat apropiado y la energía para nadar, la cual es obtenida del consumo de algas y residuos (Oliver, 1971).

\section{CICLO DE VIDA DE Chironomus sp.}

En nuestro estudio, registramos un ciclo de vida corto, con una duración de 14 días para Chironomus sp. Un ciclo de vida corto de 10 días también fue reportado para C. strenzkei (Fittkau, 1965; Danks, 2006), de 13 días (Fonseca \& Rocha, 2004) a 15 días para C. xanthus (Strixino \& Trivinho-Strixino, 1985 ) en condiciones de temperatura de $24^{\circ} \mathrm{C}$ a $26^{\circ} \mathrm{C}$. En el caso de $C$. calligrafus se registró un ciclo de vida de 23 días a $25^{\circ} \mathrm{C}$ (Florencia et al., 2008) y $C$. riparius se registró un ciclo de vida de 25 días (Jackson \& Sweeney, 1995) bajo condiciones controladas a $20{ }^{\circ} \mathrm{C}$ (Tabla 3). Muchos factores, como filogenia, disponibilidad de recursos, competencia e interferencia, temperatura, estabilidad del hábitat, entre otros pueden afectar el desarrollo de los insectos (Jackson \& Sweeney, 1995; Danks, 2006). En el caso de los Chironomus, el proceso de desarrollo larval podría ser fuertemente dependiente de la temperatura. Los reportes de ciclos de vida mencionados con menos de un mes ocurren en especies desarrolladas en ambientes con temperaturas superiores a $\operatorname{los} 20^{\circ} \mathrm{C}$. Asimismo, existen especies de quironómidos que tienen un ciclo de vida mucho más largo de hasta siete años, como aquella especie de Chironomus sp. reportada en las quebradas o lagunas de las tundras de Alaska (Butler, 1982). La distribución helicoidal en líneas paralelas de los huevos, así como el aspecto de un tubo curvado de las masas ovígeras coinciden con el patrón reportado por Fonseca \& Rocha (2004) para C. xanthus. El estadio larval de Chironomus sp. fue registrado a partir del cuarto día y tuvo una duración de ocho días, pasando por dos días adicionales en estadio de pupa. En el caso de $C$. calligrafus, Florencia et al. (2008) reportan un periodo de incubación de los huevos de uno a dos días, el período larval de 10 días, y el periodo de pupa de 10 días a temperatura de $25^{\circ} \mathrm{C}$. La superposición de las pupas antes de la emergencia de los individuos adultos en esta especie conllevó una mayor duración de esta fase. Para C. xanthus, Trivinho-Strixino y Strixino (1982) señalan que las altas temperaturas $\left(25{ }^{\circ} \mathrm{C}\right)$ fomentan el rápido desarrollo larval, favoreciendo el tiempo de emergencia del estadio adulto. Una dependencia con la temperatura se corrobora con el presente experimento, en el cual la temperatura promedio de las unidades experimentales fue de $26,4{ }^{\circ} \mathrm{C}$, registrando una emergencia de adultos a los dos días de haber comenzado la fase de pupa.

\section{CONCLUSIONES}

La producción de larvas de Chironomus sp. a través de la incubación de huevos con aireación puede ser aplicado a mayor escala con fines de producción de alimento vivo para peces en condiciones de producción y cría en acuicultura. Los huevos de quironómidos pueden ser incubados en recipientes de plástico de $1,5 \mathrm{~L}$ de capacidad, utilizando como substrato y alimento para las larvas, $250 \mathrm{ml}$ de inóculo (agua verde) y $1 \mathrm{~g}$ de harina de pescado por litro. La aireación propicia la mayor tasa de eclosión de huevos y sobrevivencia de larvas y por lo tanto esta condición de incubación debe ser preferida. La corta duración del ciclo biológico de Chironomus sp. (14 días), hace de la especie en estudio un recurso potencial y de alto valor comercial en la acuicultura. 


\section{BIBLIOGRAFÍA CITADA}

Apperson, C.; Waldvogel, M.; Bambara S. 2006. Biology and control of non-biting aquatic midges. Department of Entomology, North Carolina State University, Insect Note-ENT/rsc15, Raleigh, NC. 2 pp.

Armitage, P.D. 1995. Chironomidae as food. In: P.D. Armitage; P.S. Cranston.; L.C.V. Pinder (Editors). The Chironomidae: Biology and Ecology of Non-Biting Midges. p. 423-435.

Berg, M.B. 1995. Larval food and feeding behaviour. In Armitage, P.D.; Cranston, P.S.; Pinder, L.C.V. (eds), The Chironomidae: Biology and Ecology of Non-biting Midges. Chapman \& Hall, London: 136-168.

Butler, M.G. 1982. A 7-year life cycle of two Chironomus species in arctic Alaskan tundra ponds (Diptera: Chironomidae). Canadian Journal of Zoology, 60: 58-70.

Coffman, W.P.; Ferrington, Jr. 1979. Chironomidae. In Merrit, R.W.; Cummins, K. W. An introduction to aquatic insects of North America. 3a Ed. Department of Entomology. Michigan State University. p. 551-643.

Coffman W.P.; Ferrington, Jr.; L.C.; Berg, M.B. 2008. Chironomidae. Chapter 26 In: Merritt, R.W.; Cummins, K.W.; Berg, M.B. (Eds). An introduction to the aquatic insects of North America. 4th Ed., Kendall/Hunt Publishing, Dubuque, Iowa. p. 847-1003.

Danks, H.V. 2006. Short life cycles in insects and mites. The Canadian Entomologist. 138: 407463.

De La Noue, J.; Choubert, G. 1985. Apparent digestibility of invertebrate biomass by rainbow trout. Aquaculture, 50: 103-112.

Fittkau E.J. 1965. Revision der von E. Goeldi aus dem Amazonas gebiet beschriebenen Chironomiden (Diptera). Chironomidenstudien X. Beitrage zur Neotropischen Fauna, 4: 209226.

Florencia, L.Z.; Montalto, L.; Paggi, A.; Marchese, M.R. 2008. Biometry and life cycle of Chironomus calligraphus. Goeldi 1905 (Díptera, Chironomidae) in laboratory conditions. Interciencia, 33 (10), 767.

Fonseca, A.L.; Rocha, O. 2004. Laboratory cultures of the native species Chironomus xanthus Rempel, 1939 (Diptera-Chironomidae). Acta Limnologica Brasiliensia, 16(2): 153-161.
Habib, M.A.B.; Yusoff, F.M.; Phang, S.M.; Ang, K.J.; Mohamed, S. 1997. Nutritional values of chironomid larvae grown in palm oil mill effluent and algal culture. Aquaculture, 158: 95105.

Jackson, J.K.; Sweeney, B.W. 1995. Egg and larval development times for 35 species of tropical stream insects from Costa Rica. Journal of the North American Benthological Society, 14: 115130.

McLachlan, A.J.; Pearce, L.J.; Smith, J. A. 1979. Feeding interactions and cycling of peat in a bog lake. Journal of Animal Ecology, 48: 851-861

Mason, W.T. 1973. An introduction to the identification of chironomid larvae. Analytical Quality Control laboratory National Environmental Research Center U.S. Environmental Protection Agency. Cincinnati, 98pp.

Mclarney, W.O.; Henderson, S. Sherman, M.M. 1974. A new method for culturing Chironomus tentans Fabricius. Larvae using burlap substrate in fertilized pools. Aquaculture, 4: 267-276.

Navarrete, N.; Fernández, G.; Contreras, G. 2004. Abundancia de Quironomidos (Díptera: Chironomidae) en el bordo "JC" del norte del estado de México en periodo de secas. Hidrobiológica, 14(2): 157-160.

Oliver, D.R. 1971. "Life history of the Chironomidae." Annual review of entomology, 16.1:211-230.

Pinder, L.C.V. 1986. Biology of freshwater Chironomidae. Annual Review of Entomology, 31:1-23.

Pinder, L.C.V. 1995. The habitats of chironomid larvae. In: The Chironomidae: biology and ecology of non-biting midges. P.D. Armitage, P.S. Cranston \& L.C.V. Pinder (eds.) p. 107-135.

Strixino, G; Trivinho-Strixino, S. 1985. A temperatura e o desenvolvimento larval de Chironomus sancticaroli (Diptera: Chironomidae). Revista Brasileira de Zoologia, 3: $177-180$.

Tidwell, J.H.; Schulmeister, C.M.; Coyle, s. 1997. Growth, survival, and Biochemical Composition of freshwater prawns Macrobrachium rosenbergii fed Natural food organisms under controlled conditions. Journal of the World Aquaculture Society, 28: (2), 123-132. 
Sugden, L.G. 1973. Feeding ecology of pintail, gadwall, American widgeon, and lesser scaup ducklings. Canadian Wildlife Service. 24. 45pp.

Trivinho-Strixino, S.; Strixino, G. 1982. Ciclo de vida de Chironomus sancticaroli Strixino and Strixino, (Diptera, Chironomidae). Revista Brasileira de Entomologia, 26: 183-189.

Trivinho-Strixino, S. 2011. Larvas de Chironomidae. Guía de identificaçâo. Sâo
Carlos, Depto. Hidrobiología/Lab. Entomologia Aquâtica/UFSCar. 371 p.: Il., estampas.

Ward, A.F.; Williams, D.D. 1986. Longitudinal zonation and food of larval chironomids (Insecta: Diptera) along the course of a river in temperate Canada. Ecography, 9(1), 48-57.

Recibido: 4 de Febrero del 2016

Aceptado para publicación: 4 de Abril del 2016 